Annuaire suisse de politique de développement

$14 \mid 1995$

Annuaire Suisse - Tiers Monde 1995

\title{
Cohérence et cohésion dans la coopération au développement
}

Günther Baechler

\section{(2) OpenEdition}

1 Journals

Édition électronique

URL : http://journals.openedition.org/aspd/1448

DOI : $10.4000 /$ aspd. 1448

ISSN : 1663-9669

Éditeur

Institut de hautes études internationales et du développement

Édition imprimée

Date de publication : 1 février 1995

Pagination : 174-176

ISSN : 1660-5934

\section{Référence électronique}

Günther Baechler, «Cohérence et cohésion dans la coopération au développement », Annuaire suisse

de politique de développement [En ligne], 14 | 1995, mis en ligne le 03 mai 2013, consulté le 08

septembre 2020. URL : http://journals.openedition.org/aspd/1448 ; DOI : https://doi.org/10.4000/

aspd. 1448

(C) The Graduate Institute | Geneva 


\title{
Cohérence et cohésion dans la coopération au développement
}

\author{
Günther Bächler, Fondation suisse pour la paix
}

\section{Les problèmes de la cohérence dans les relations de la Suisse avec I'Indonésie}

Le Conseil fédéral a déterminé cinq objectifs de politique extérieure qui sont non seulement l'expression normative d'une prise en compte cohérente des intérêts de la Suisse mais qui doivent permettre d'obtenir dans le pays concerné autant que possible l'effet escompté: paix et sécurité, droits de l'homme, assistance sociale, élimination des discriminations sociales, sauvegarde des bases naturelles de l'existence.

II existe fondamentalement deux méthodes différentes pour mesurer la cohérence d'une politique. D'une part, on mesure la cohérence chez l'instigateur de cette politique, par exemple, en réunissant les divers groupes d'intérêt, afin d'obtenir le meilleur équilibre possible entre les cinq objectifs, entre les différents intérêts, ainsi qu'entre les cinq objectifs et intérêts. D'autre part, on mesure la cohérence chez le bénéficiaire de cette politique, en essayant d'établir si cette politique a atteint ou non le but escompté. Dans l'article ciaprès, j'aimerais me concentrer sur le bénéficiaire.

Si l'on voulait mesurer la cohérence d'une politique aux effets concrets qu'elle entraîne chez le bénéficiaire, on pourrait choisir comme indicateur la cohésion socio-culturelle. J'entends par là (à l'instar de Verena Tobler Müller) la cohésion interne d'une culture, l'intégration fonctionnelle et l'interpénétration de la technologie et de l'organisation sociale, sans oublier un gouvernement et une administration légitimés et acceptés. Je pars de l'hypothèse selon laquelle une cohésion relativement élevée qui prévaut dans une société permet la réalisation des cinq objectifs du Conseil fédéral. A l'inverse: si la cohésion est faible, les conflits politiques se développant dans les domaines concernés par les cinq objectifs sont plus nombreux et plus exacerbés dans une société.

Question force de cohésion, on peut dire que celle de l'Indonésie est en déclin. Le pays est en effet traversé par de nombreux conflits qui menacent aussi bien l'intégration sociale que l'intégration politique (de l'État). De ce fait, la cohérence politique du côté de la Suisse (et d'autres partenaires) s'en trouve altérée et pourrait même devenir dépassée à l'avenir. Pour n'en citer que quelques-uns:

- le fossé grandissant entre le clan de Suharto et une couche moyenne de la population qui ne cesse de croître sur le plan numérique; 
- les lignes de scission à l'intérieur de l'élite qui ne feront que s'approfondir au fur et à mesure que l'échéance d'un changement de pouvoir se rapprochera;

- la structure dualiste de la société indonésienne qui se reflète dans l'antagonisme croissant entre la ville et la campagne, la métropole et la périphérie;

- l'oppression violente de l'indépendance d'Irian Jaya (et de l'OPM);

- le combat contre le Front de libération nationale Aceh Sumatra;

- le déplacement de la population dans le cadre du programme de transmigration;

- la répression de toute opposition politique depuis les massacres du début des années soixante;

- les antagonismes socio-économiques qui caractérisent le secteur moderne et qui conduisent à une agitation croissante dans le monde du travail;

- l'abandon d'une agriculture solide et durable comme base de développement pour l'ensemble du pays et surtout

- la crise de l'agriculture vivrière qui contribue à la marginalisation d'au moins un cinquième de la société indonésienne.

Du fait de la stabilité relative du régime et de sa supériorité militaire par rapport aux mouvements de libération, les conflits sont contenus; cela ne veut toutefois pas dire que l'on en ait supprimé le germe. La structure dualiste de l'Indonésie favorise unilatéralement le secteur moderne au détriment du secteur traditionnel, le secteur agricole. On observe à cet égard deux phénomènes: une "marginalisation de la pauvreté" (20 - 27\% de la population) aux zones périphériques de l'Indonésie et aux nombreux "secteurs nationaux sacrifiés" (industrie minière, Mine Ok-Tedi, déboisement, etc.). Malgré l'assimilation forcée appliquée à l'est de l'île de Timor et à l'Irian Jaya - et qui entraîne des investissements considérables dans le système éducatif - l'unité ethnique, religieuse, socioculturelle et linguistique demeure un leurre sur cet archipel. A cela s'ajoute le fait que les deux conflits mentionnés - qui se caractérisent par leur asymétrie - sont de longue haleine, douloureux et coûtent une fortune. Le programme de transmigration envenime dans une large mesure les conflits entre le gouvernement central et les populations des différentes îles - notamment dans l'ouest de l'Irian.

La répression de toute opposition politique souligne le fait que l'amélioration des conditions socio-économiques des 20 dernières années s'explique davantage par le maintien d'une "dictature du développement" que sur la démocratie et un régime de "Good Governance". Les confrontations sociales qui agitent le secteur moderne sont au demeurant les signes d'une inadéquation croissante du style de pouvoir actuel. Les exigences de participation ne peuvent être satisfaites, tandis que les chances d'élévation sociale sont, elles aussi, relativement réduites à cause de la carence en emplois salariés et d'une formation professionnelle insuffisante. Dans le World Development de la Banque Mondiale, I'Indonésie figure encore aujourd'hui comme Low Income Economy. 


\section{Dissolution des contradictions et des mécanismes actuels}

Une politique extérieure cohérente doit contribuer à atténuer les conflits et à stimuler la force de cohésion. Des mesures positives - comparables aux délégations de pouvoir dans le cadre national - doivent viser à favoriser la suppression des antagonismes sociaux et régionaux et à rendre plus perméables les frontières entre les deux secteurs. II faudra en particulier que l'on mesure la cohérence de la politique à la valeur qu'elle accordera au développement de l'agriculture. Si l'on peut partir de l'hypothèse selon laquelle, dans les pays en développement - mais aussi dans les pays limitrophes et ceux du tigre Sud-Est asiatique - la majorité des gens vivent à la campagne, il faudra donc accorder une plus grande attention à ce secteur. On sait parfaitement aujourd'hui que le mépris du secteur agricole constitue l'un des principaux facteurs susceptibles de provoquer, à long terme, une crise du développement. Les conséquences de la crise sont de plus en plus souvent illustrées par les problèmes écologiques qui accentuent pour leur part la crise sociale et entravent, de surcroît, la transition démographique à des contingents de population stables. Tôt ou tard, une crise qui se développe simultanément à plusieurs niveaux - social, écologique et démographique - finira par toucher également le secteur moderne. Une politique offensive de modernisation ne suffit pas à elle seule pour résoudre ces problèmes, pour ainsi dire, "à la remorque".

Pour les investisseurs privés de l'étranger, cet ensemble de problèmes constitue un grave défi. Certes, cela n'empêche pas de faire des bénéfices à court et à moyen terme et les entreprises étrangères peuvent de nouveau se retirer lorsque les crises prennent de l'ampleur - comme c'est actuellement le cas au Nigéria. Mais est-ce bien là une politique cohérente?

\section{Analyse de "compatibilité avec la paix" comme instrument d'une politi- que cohérente}

Une politique qui ne mise que sur le secteur moderne et favorise une évolution unilatérale et fortement centralisée ne peut guère être qualifiée de "cohérente". D'où la nécessité pour, en particulier, l'administration et l'industrie privée de s'entendre avec les syndicats, les associations d'entraide et les groupes luttant pour les droits de l'homme sur ce qui favorise la cohésion et sur ce qui la remet en cause.

Une "analyse de compatibilité avec la paix" de sa propre manière d'agir qui devrait être effectuée à travers le dialogue avec les différents acteurs et qui tienne compte des domaines de conflit susmentionnés pourrait être l'instrument permettant de mettre en corrélation les efforts de cohérence de l'instigateur de cette politique avec les efforts de cohésion de son bénéficiaire. II faudra en outre réfléchir, dans le cadre d'une autre étude, aux indicateurs et mécanismes que doit comporter un tel examen de la compatibilité d'une politique cohérente avec la paix. 